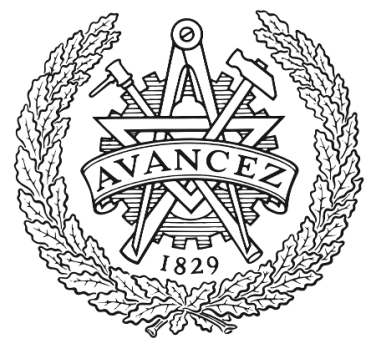

CHALMERS

UNIVERSITY OF TECHNOLOGY

\title{
Superchannel Engineering with Microresonator Combs
}

Downloaded from: https://research.chalmers.se, 2023-04-26 10:03 UTC

Citation for the original published paper (version of record):

Helgason, Ò., Fülöp, A., Schröder, J. et al (2018). Superchannel Engineering with Microresonator

Combs. 2018 Conference on Lasers and Electro-Optics, CLEO 2018.

http://dx.doi.org/10.1364/CLEO_AT.2018.JW2A.71

N.B. When citing this work, cite the original published paper. 


\title{
Superchannel Engineering with Microresonator Combs
}

\author{
Óskar B. Helgason ${ }^{1, *}$, Attila Fülöp1, Jochen Schröder ${ }^{1}$, Peter A. Andrekson¹, Andrew M. Weiner ${ }^{2}$ and \\ Victor Torres-Company ${ }^{1}$ \\ ${ }^{I}$ Photonics Laboratory, Department of Microtechnology and Nanoscience, Chalmers University of Technology, SE-41296 Göteborg, Sweden \\ ${ }^{2}$ School of Electrical and Computer Engineering, Purdue University, West Lafayette, IN 47907-2035, USA \\ *skarb@chalmers.se
}

\begin{abstract}
We study segmenting the available bandwidth of a WDM system into microcombdriven superchannels. This solution improves the power per line while using a fraction of the pump power, making it potentially more favorable for integration.

OCIS codes: (190.4390) Nonlinear optics, integrated optics; (060.1660) Coherent communications
\end{abstract}

\section{Introduction}

Microresonator-based optical frequency combs constitute an emerging chip-scale technology for wavelengthdivision-multiplexed (WDM) communication systems [1-3]. Temporal bright solitons have a smooth optical spectrum and the line spacing and bandwidth can be precisely controlled with the dimensions of the cavity [1,4]. Recent experiments indicate that bright soliton combs can generate hundreds of high-quality comb lines on chip with a performance in terms of optical signal-to-noise ratio (OSNR) sufficient to encode complex modulation formats [1], yielding astonishingly high data rates.

A drawback with the generation of bright temporal solitons for massively parallel coherent communications is that the conversion efficiency (the ratio between generated comb power to pump power) scales with $\sim 1 / N$, where $N$ is the number of generated comb lines [4]. Hence, the power of each comb line will scale with $\sim 1 / N^{2}$. Since the OSNR of each line is limited by its power and the quantum noise floor, this type of experiment requires a tunable high-power $(\sim 1 \mathrm{~W})$ laser to achieve the needed OSNR for coherent optical communications [1].

Here, instead of attempting to cover the full $10 \mathrm{THz} \mathrm{C}+\mathrm{L}$ band with a single comb source, we propose using a superchannel approach [5]. The $10 \mathrm{THz}$ band is divided into superchannels, each fed by a comb source (see Fig. 1). According to the scaling law in nonlinear conversion efficiency [4], this solution relaxes the pump requirement to each superchannel by a factor equal to the number of superchannels and increases in the same proportion the power per line. We additionally investigate the same approach with dark pulse combs [6], which have a higher conversion efficiency than the bright solitons [7]. We demonstrate that dividing the bandwidth into narrower superchannels has indeed a superior performance compared to the single comb source, for both bright soliton and dark pulse combs.

a) Single comb source

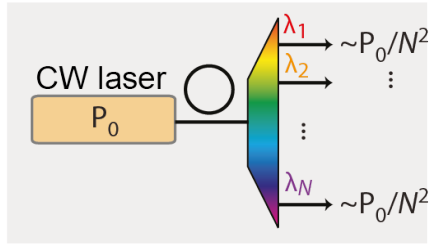

b) Several superchannels

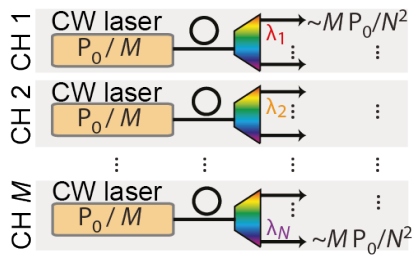

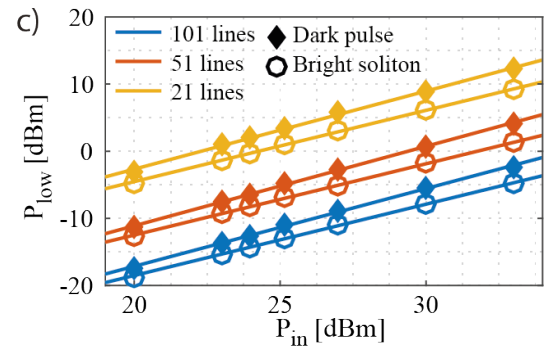

Fig. 1: The diagram in a) shows the single comb approach, providing $N$ lines using a single comb and CW laser with pump power $\mathrm{P}_{0}$. In $\mathrm{b}$ ) the total optical power has been distributed between $M$ lasers, each pumping a separate comb providing $N / M$ lines. According to the nonlinear scaling law this solution yields $M$ times more power per line than the single comb. The graph in c) shows the simulated performance of individual combs generating $N$ lines at different pump power, where $P_{\text {low }}$ is the lowest power among these $N$ lines. Each comb in the plot is found optimally in terms of group velocity dispersion, coupling rate and detuning. If we pick two combs that fit the approach in a) and b), e.g. 1 comb with 101 lines generated at $P_{\mathrm{in}}=30 \mathrm{dBm}$ compared to $M=5$ combs with 21 lines generated at $P_{\mathrm{in}}=23 \mathrm{dBm}$, we see an improvement in performance by a factor $M$.

\section{Superchannel engineering with microresonator frequency combs}

Superchannels have previously been used to overcome the electrical data rate limits of transmitters by grouping several modulated coherent optical lines into one channel [5]. By segmenting the available bandwidth into smaller superchannels, one gains in flexibility, since each superchannel can feature different line spacing with different modulation formats. This solution also allows using comb sources in optical networks where dynamic routing using add/drop multiplexers is needed. Superchannels benefit from the line spacing stability provided by frequency comb sources to achieve high spectral efficiency [5].

Let us compare the situation sketched in Fig. $1 \mathrm{a}-\mathrm{b})$. In a), we have a single laser source with power $\mathrm{P}_{0}$ pumping 
a microresonator designed to yield $N$ lines with a certain spacing covering a target bandwidth. The total comb output power will be $\sim \mathrm{P}_{0} / N$, thus leading to a power per line $\sim \mathrm{P}_{0} / N^{2}$. Consider instead the situation in $\mathrm{b}$ ), where a laser with power $\mathrm{P}_{0} / M$ pumps a different microresonator that is designed to provide $N / M$ lines while keeping the same line spacing. $M$ superchannels would cover a bandwidth equivalent to the first case and use the same total pump power (although it would require $M$ lasers). Owing to the nonlinear conversion efficiency, the second case is much more power efficient: it yields $M$ times higher power per line while using the same total pump power. In the next section, we verify this favourable superchannel scaling via numerical simulations.
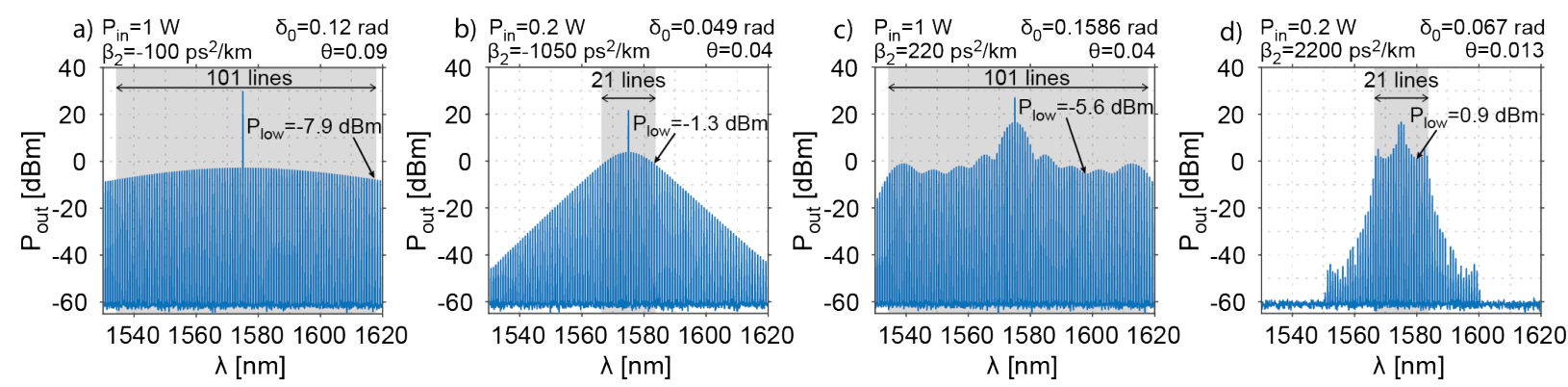

Fig. 2: a-b) show the optimal bright pulse combs for pump powers $1 \mathrm{~W}$ and $0.2 \mathrm{~W}$, providing 101 lines and 21 respectively. c-d) show the optimal dark combs for the same number of lines and pump power as in $a-b$ ). One can deduce from the figures that using five 21 line combs (producing 105 lines) will result in a higher power per line than using a single 101 line comb, while the total optical pump power is the same in both cases.

\section{Numerical verification}

To evaluate the suggested superchannel architecture, we compare comb cases with different pump powers $\left(P_{\text {in }}\right)$, providing 101, 51 and 21 carriers, for both dark and bright pulse combs with FSR=100 GHz. Assuming that the comb lines have to be equalized to the level of the lowest power line, we use the power of the lowest line among the carriers, $P_{\text {low }}$, as a figure of merit. We map the performance through numerical simulations and find the best performing comb of every case in terms of group velocity dispersion, $\beta_{2}$, coupling rate, $\theta$, and detuning, $\delta_{0}$. The losses, the group index and nonlinear parameter are set to $\alpha=0.1 \mathrm{~dB} / \mathrm{cm}\left(Q_{\mathrm{int}}=3.5\right.$ million $), n_{\mathrm{g}}=2$ and $\gamma=2 \mathrm{~m}^{-1} \mathrm{~W}^{-1}$. Higher order effects are assumed to be negligible. The comb states are simulated using the Ikeda map [8], where noise (in this case quantum fluctuations) can be added with the input pump [9]. The combs are initiated with either a hyperbolic secant or a dark square pulse for bright and dark pulse combs respectively $[4,6]$.

The results are plotted in Fig. $1 \mathrm{c}$ ). The figure shows that the power per line grows almost linearly with pump power. Furthermore, there is a $\sim 6 \mathrm{~dB}$ increase in power per line when the number of lines is halved, indicating the forementioned $\sim 1 / N^{2}$. This scaling applies both for dark and bright pulse combs, showing that the scaling laws in ref. 4 also apply to dark pulse combs, where the dark pulse combs perform $\sim 2.3 \mathrm{~dB}$ better. To complete our investigation, we compare the case of a single comb pumped with $1 \mathrm{~W}$ providing 101 lines with 5 superchannels pumped with $0.2 \mathrm{~W}$ providing 21 lines each. The resulting combs are plotted in Fig 2. Comparing the 21 line combs with the 101 line combs, an improvement in power per line of roughly $6.5 \mathrm{~dB}$ is seen for both the bright soliton and dark pulse case, despite using 5 times lower power. This is close to estimated improvement of $M=5$ in the previous section.

In summary, we have proposed a chip-scale transmitter architecture based on microresonators with a $10 \mathrm{THz}$ bandwidth that consists of five superchannels covering $2 \mathrm{THz}$ each. Compared with single comb sources covering the full bandwidth, we have found that using this approach increases the minimum line power drastically, leading to power per line improvements of $\sim 6.5 \mathrm{~dB}$ for both bright soliton and dark pulse combs. Additionally, since the pump power is decreased by a factor of five, the requirement of an initial high-power EDFA is diminished. We envision microresonator-based combs as light sources in integrated transmitters for modern superchannel-based systems.

\section{References}

[1] P. Marin-Palomo et al., "Microresonator-based solitons for massively parallel coherent optical communications," Nature 546, 274 (2017).

[2] A. Fülöp et al., "Long-haul coherent communications using microresonator-based frequency combs," Opt. Express 25, 26678 (2017).

[3] J. S. Levy et al., "CMOS-compatible multiple-wavelength oscillator for on-chip optical interconnects," Nat. Photonics 4, 37-40 (2010).

[4] C. Bao et al., "Nonlinear conversion efficiency in Kerr frequency comb generation," Opt. Lett. 39, 6126 (2014).

[5] S. Chandrasekhar and X. Liu, "Advances in Tb/s superchannels," in Optical Fiber Telecommunications VIB (2013).

[6] X. Xue et al., "Mode-locked dark pulse Kerr combs in normal-dispersion microresonators," Nat. Photonics 9, 594-600 (2015).

[7] X. Xue et al., "Microresonator Kerr frequency combs with high conversion efficiency," Laser Photon. Rev. 11, 1600276 (2017).

[8] K. Ikeda, "Multiple-valued stationary state and its instability of the transmitted light by a ring cavity system," Opt. Commun. 30, 257 (1979).

[9] V. Torres-Company et al., "Comparative analysis of spectral coherence in microresonator frequency combs," Opt. Express 22, 4678 (2014).

We gratefully acknowledge funding support from the Swedish Research Council 